\title{
High-Precision Time-of-Arrival Estimation for UWB Localizers in Indoor Multipath Channels
}

\author{
Marzieh Dashti ${ }^{1}$, Mir Ghoraishi $^{1}$, Katsuyuki Haneda ${ }^{2}$ and Jun-ichi Takada ${ }^{1}$ \\ ${ }^{1}$ Tokyo Institute of Technology \\ ${ }^{2}$ Aalto University School of Science and Technology \\ ${ }^{1}$ Japan \\ ${ }^{2}$ Finland
}

\section{Introduction}

The global positioning system (GPS) has found application in many different fields, in areas where there is a good line-of-sight (LoS) to GPS satellites, this technique provides a good estimate of the location of user terminal (UT). However, in indoor and dense urban environments, localization has always been a more challenging problem for several reasons. Typically the GPS signal is not strong enough to penetrate through most materials. As soon as an object obscures the GPS satellite from the UT's view, the signal is corrupted. This constrains the usefulness of GPS to open environments, and limits its performance in forests or in dense urban environments, as retaining a lock on the GPS signals becomes more difficult. GPS typically becomes completely useless inside buildings. However there is an increasing need for accurate localization in cluttered environments, in addition to open spaces. In commercial applications for example, the tracking of inventory in warehouses or cargo ships is an emerging need. In military applications the problem of blue force tracking, i.e., knowing where friendly forces are, is of vital importance. This is not a problem in open environments where systems can rely on GPS, but in dense urban or indoor environment, no satisfactory solution exists. Navigation in GPS-denied environment is also a pressing military need. For example untethered robots operating in enclosed environments such as urban canyons or inside buildings need accurate positioning to safely navigate. Indoor localization is of great importance for the applications that a person or a vehicle enter a building and accurately tracking its position over time is needed and the position estimate should have a precision of under one meter, i.e. on the order of some of the building feature dimensions, such as hallway width.

\subsection{Indoor localization}

To address the problem of localization in cluttered environments, deploying a wireless sensor network (WSN) composed of fixed sensors emitting radio signals is considered in (Jourdan, 2006). Once sensors are deployed, the location of them is known. For example the sensors can be placed outside and rely on GPS, or UTs can place them inside and determine their locations by survey or other means (e.g. an accurate map). The UT can then extract range estimates to the sensors from the received signals (for instance by time-of-arrival (ToA) estimation), and then use the range estimates in a triangulation technique to determine its own 


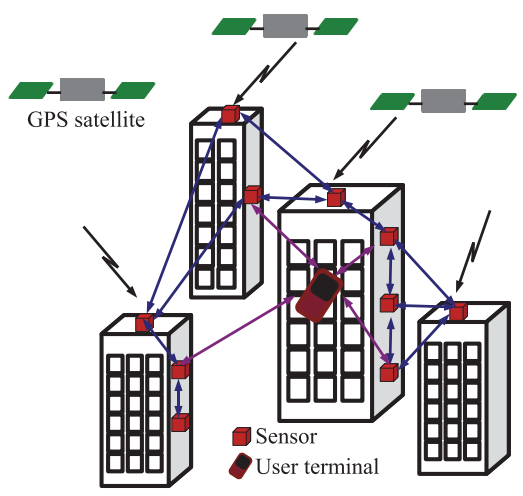

Fig. 1. Indoor localization architecture

position. Among various approaches (Falsi et al., 2006; Gezici et al., 2005; Lee \& Scholtz, 2002; Low et al., 2005), use of ultra-wideband (UWB) signal is a promising technology in particular for indoor application. The sensors transmit UWB signals in the case, which own inherent delay resolution and ability to penetrate obstacles (Jourdan, 2006; Win \& Scholtz, 1998; 2002). Further information on the fundamentals of UWB can be found in (Jourdan, 2006) and the references therein. The indoor localization architecture is illustrated on Fig. 1. Assume an emergency scenario as an example application of localization, in response to an emergency in a high rise building, a network of UWB sensors is deployed. These sensors provide a localization network to responders (or UTs) moving inside the building. The role of the sensors is to provide the UT with ranges. The UT would broadcast a UWB signal, so that the sensors each measure the range to the UT, share the information and infer the location of the UT. The quality of range measurements will degrade with distance, so that the distance between sensors is of concern. The ranging algorithm can also easily accommodate range constraints between sensors and UTs (Jourdan, 2006). The described localization architecture is called user terminal based localization technique, i.e. the localization task is performed by the UT itself and the sensors do not need to interact with one another to perform the localization task. The UT-based technology requires the installation of client software on the UT to determine its location. On the other hand, network-based techniques utilize the service provider's network infrastructure to identify the location of the UT. The advantage of network-based techniques is that they can be implemented non-intrusively, without affecting the UTs. One of the key challenges of network-based techniques is the requirement to work closely with the service provider, as it entails the installation of hardware and software within the operator's infrastructure. Often, a legislative framework, such as E911, would need to be in place to compel the cooperation of the service provider as well as to safeguard the privacy of the information. The focus of this chapter is exclusively on the range estimation between UT and AP sensors, and the network architecture is not discussed in remainder of this chapter. This implies that issues related to the communication connectivity between sensors, etc., are not presented. Without lost of generality of ranging analysis, any of UT-based or network-based localization systems can be assumed. 


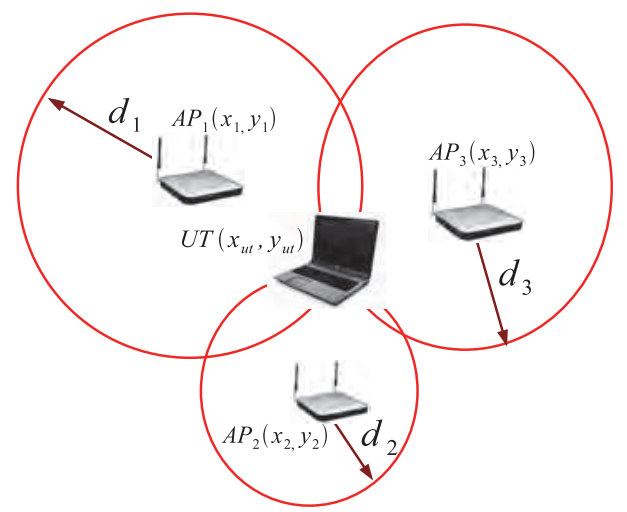

Fig. 2. Time-of-arrival triangulation of ranges to determine location

\subsection{ToA-based ranging}

An appropriate method for the indoor ranging problem is based on timing. Assuming the sensors and the UT are synchronized in time, the UT can calculate the time of arrival (time-of-flight) of a signal by comparing its time stamp at transmission to its ToA. This can then be converted to a distance by multiplying the time-of-flight by the speed of light. Since the accuracy of ToA estimation increases with the signal-to-noise ratio (SNR) and the bandwidth (BW) (Gezici et al., 2005), UWB (for a given SNR) will typically achieve great ToA accuracy compared to narrower band signals. Note that in general the sensors and the UT will not have a common time reference, in which case variants of this method must be used. In the round-trip method, the UT transmits a UWB signal to a certain sensor (IEEE Std, 2007). Once it is received at the sensor, it is retransmitted and in turn received by the UT. By comparing the time of original transmission to the ToA (and accounting for the processing time required for the sensor to retransmit the signal), a time-of-flight can be determined (Lee \& Scholtz, 2002). In 2D, three sensors are sufficient to generate a position estimate. Fig. 2 shows the ToA triangulation of ranges to determine location. The technique assumes that three (or more) ranges $c \times t_{1}, c \times t_{2}$ and $c \times t_{3}$ (where $c$ is speed of light) are gathered from APs $i=1,2$, and 3 , respectively, with known locations $\left(x_{i}, y_{i}\right)$, as in Fig. 2. Solving the set of equations of

$$
\left\{\begin{array}{l}
c \times t_{1}=\sqrt{\left(x_{1}-x_{u t}\right)^{2}+\left(y_{1}-y_{u t}\right)^{2}} \\
c \times t_{2}=\sqrt{\left(x_{2}-x_{u t}\right)^{2}+\left(y_{2}-y_{u t}\right)^{2}} \\
c \times t_{3}=\sqrt{\left(x_{3}-x_{u t}\right)^{2}+\left(y_{3}-y_{u t}\right)^{2}}
\end{array}\right.
$$

translates to finding the intersection of the three circles (or spheres in three dimensions), yielding the unknown coordinates of the UT. Time of arrival is appealing due to its application to the general network architecture, however, the associate asynchronous ranging requires two or more messages per ranging session. This requirement may potentially increase the network traffic considerably (Jourdan, 2006). If the AP sensors are synchronized, but not the UT, then the time-of-difference-of-arrival (TDoA) method can be used. In this case the UT transmits a UWB signal, and the TDoA is computed at two sensors. The UT is then located on a hyperbola with foci at the sensors. 
In the remainder of the chapter, for simplicity and without loss of generality, we assume that the sensors and the UT have a common time-reference.

\subsection{Challenges to UWB ranging}

Let's refer to a range measurement between a transmitter $(T x)$ and a receiver $(\mathrm{Rx})$ as a direct path (DP) measurement if the range is obtained from the signal traveling along a straight line between the two points. Range measurements are typically corrupted by multipath fading, thermal noise, DP blockage, and DP excess delay. Multipath fading is due to destructive and constructive interference of signals at the receiver arriving via different propagation paths. This makes the detection of DP, if present, challenging. When the received signals are from reflections, resulting in measured ranges larger than the true distances. The difficulty is due to DP excess delay incurred by propagation of the partially obstructed DP through different materials, such as walls. When such a partially obstructed DP signal is observed as first arrival, the propagation time depends not only upon the traveled distance, but also upon the materials it encountered. Because the propagation of electro-magnetic signals is slower in some materials than in the air, the signal arrives with excess delay, again yielding a range estimate larger than the true one. The effect of DP blockage and DP excess delay is the same: they both add a positive bias to the true range between UT and sensor, so that the measured range is larger than the true value. This positive error has been identified as a limiting factor in UWB ranging performance (Falsi et al., 2006; Lee \& Scholtz, 2002), so it must be accounted.

\subsection{Contribution of the chapter}

This chapter reviews the ToA estimation algorithms and then employs a threshold-based ToA estimation algorithm to calculate the range between $\mathrm{Tx}$ and $\mathrm{Rx}$ nodes in an indoor multipath environment. A practical threshold setting technique is introduced. For the purpose of this study, a set of empirical data obtained to create a baseline for comparative performance evaluation of ranging algorithms. The measured ranging error is used as a criteria to evaluate the ToA estimation algorithm.

\section{UWB ToA estimation}

As described in previous section, ToA estimation technique used with UWB transmission can be used for accurate indoor ranging. The transmitter sends out a UWB ranging signal $\sqrt{E_{\mathrm{tx}}} p_{\mathrm{tx}}(t)$ where $p_{\mathrm{tx}}$ is the monocycle pulse waveform with normalized energy after passing through a root-raised cosine bandpass filter with bandwidth, BW, adopted from (IEEE Std, 2007), and $E_{\mathrm{tx}}$ is the pulse energy. Standard UWB pulse with BW=0.5GHz is shown in Fig. 3. The received UWB signal in multipath channel is represented as

$$
r(t)=\sum_{i=0}^{I} \sqrt{E_{\mathrm{tx}}} \alpha_{i} p_{i}(t)+w(t)
$$

where $\alpha_{i}$ is the complex path-gain of the $i$ th path assuming $I+1^{\prime}$ effective multipaths in the channel, and $w(t)$ is zero mean Gaussian random noise with variance $\sigma_{w}^{2}$. In the LoS scenario the first arriving multipath is the direct path and the remaining I multipaths arrive to the receiver after one or more interactions (scattering, reflection, diffraction) in the channel. It is known that the UWB waveform is distorted during interactions to the wireless channel. A simplifying assumption is to consider this distortion negligible, i.e. $p_{i}(t)=p_{\mathrm{tx}}\left(t-\tau_{i}\right)$ with $\tau_{i}=l_{i} / c$ representing the delay of the $i$ th multipath, $c$ is the 


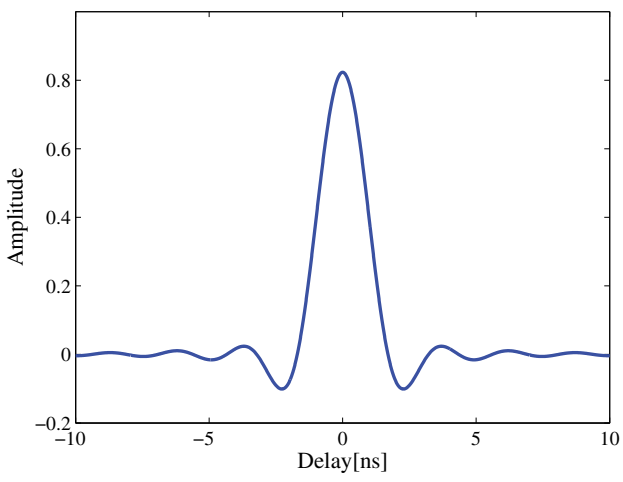

Fig. 3. Standard UWB pulse, BW=0.5GHz.

propagation velocity of electromagnetic wave and $l_{i}$ is the path length, i.e. the distance $i$ th path travels from transmitter to receiver. A comparative study of different UWB transceivers employed for ToA estimation is presented in (Guvenc et al., 2006). Matched-filter (MF) receiver is known to obtain better decision for ToA estimation than the energy-detector (ED) however it requires the knowledge of the received pulse shape which may not be available in practice (Sahinoglu et al., 2003). Another disadvantage of the MF is the requirement of Nyquist rate sampling for accurate ranging, hence complex analogue-to-digital converters are required. On the other hand ED based estimator is preferable due to its low complexity, e.g. implementation at sub-Nyquist sampling rates (Sahinoglu et al., 2003). A disadvantage for the ED is the squared noise component at its output which makes a poor performance in low SNR scenarios. The ED output samples can be represented as

$$
z(n)=\int_{n T}^{(n+1) T}|r(t)|^{2} d t
$$

where $T$ is sampling period, $n \in 1, \ldots, N$ is sample index and $N$ represents the total number of received samples. The integrator performs the sampling operation by successively integrating the squared received signal. First arriving sample is presented by $n_{0}$ which in the LoS scenario corresponds to the direct path with the propagation delay $\tau_{0}$. If the transmitter and receiver are by some means synchronized their range $d_{0}$ can then be obtained from the relation $d_{0}=$ $c \tau_{0}$.

\subsection{Review of UWB ToA estimation algorithms}

Several methods for ToA estimation of UWB signals can be found in (Falsi et al., 2006; Gezici et al., 2005; Low et al., 2005), i.e., examples of maximum likelihood based ranging, low-complexity estimators include a maximum peak detection method and a threshold detection method. In the latter a threshold is chosen a priori, and the ToA is defined as the instant when the received amplitude goes above this threshold (Falsi et al., 2006). In spite of its simplicity, this method works quite well for UWB signals, especially in high SNR environments. In this chapter the performance of method is further analyzed by details. 


\subsubsection{Maximum likelihood based ranging}

Range estimation based on Maximum likelihood (ML) techniques is explained (Sahinoglu et al., 2003). Starting from the assumption of full a-priori information, various degrees of assumptions about the unknown parameters are considered (Gezici et al., 2005), (Falsi et al., 2006), (Win \& Scholtz, 2002). In addition, the generalized ML ratio test approach and the sub-Nyquist sampling ML approaches are discussed (Win \& Scholtz, 1998),(Guvenc \& Sahiinoglu, 2005). ML ToA estimation (equivalently, ranging) can be performed by means of a correlator with a template signal that is perfectly matched to the received multipath signal, and by determining the time delay of the template for which the correlator output is maximized (Gezici et al., 2005). That optimal template can be expressed as

$$
p_{\mathrm{tmp}}(t)=\sum_{i=0}^{I} \alpha_{i} p_{i}\left(t-\tau_{i}\right)
$$

However, this ML receiver cannot be implemented in practice, since the received waveform has unknown parameters to be estimated, i.e., delays, amplitudes, and pulse shapes of different multipath components (MPCs)(Guvenc et al., 2008). A ML Estimation with no prior information is described in (Guvenc et al., 2008), which in this method, the ML estimation of the delays and the channel coefficients has high computational complexity as it requires a search over different values of delays. As explained, the ML estimation based on the channel estimation problem provides an optimal ToA estimate. However, it is quite impractical for UWB channels with a large number of MPCs as the estimation of all the channel parameters is computationally very intensive. Therefore, a simplified version of the ML approach, called generalized maximum likelihood (GML) technique, is studied in (Win \& Scholtz, 1998). Assuming that the strongest MPC has been detected, the GML technique estimates the ToA by performing a search over a smaller search space. Specifically, the paths prior to the strongest MPC are investigated. In (Win \& Scholtz, 1998), an iterative technique with lower complexity is proposed which uses certain prior statistics of the UWB channel to define a new search space and to set a stopping rule. The ML techniques can be impractical in many cases due to their computational complexity and high sampling rate requirements. In (Guvenc et al., 2008), ToA estimators that operate at low sampling rates and with various levels of a-priori information are described. These methods require prior information related to the statistics of individual ED samples, which, such prior information may not be available in practice.

\subsubsection{Low complexity ranging techniques}

In this section, peak detection algorithms (Falsi et al., 2006), two step TOA estimation approaches, ranging with dirty templates (Yaung \& Giannakis, 2004), (Yaung \& Giannakis, 2005), and threshold based ranging algorithms (Dardari \& Win, 2006)-(Scholtz \& Lee, 2002) are discussed.

- Peak detection techniques The maximum energy selection (MES) approach is a simple technique that estimates the ToA according to the strongest sample. It can be formulated as

$$
\begin{aligned}
\tau_{\mathrm{D}} & =n_{\mathrm{D}} T, \\
n_{\mathrm{D}} & =\underset{n}{\operatorname{argmax}}(z[n]),
\end{aligned}
$$

where $\tau_{\mathrm{D}}$ is detected sample as direct path. In typical UWB channels, the number of samples which include both desired signal and noise can be much larger than 1 when 


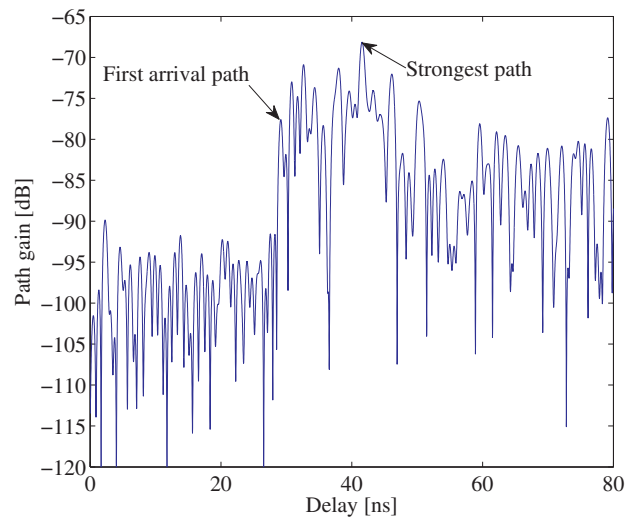

Fig. 4. An example of CIR where the FAP is not the same as SP.

the sampling interval is on the order of a pulse duration. Therefore, there can be a large delay between the first path and the strongest path, and the MES technique can yield large estimation errors. In the other hand, the strongest path (SP) in many cases even in LoS scenario is not the first arrival path (FAP) as shown in figure 4. In ranging analysis, FAP should be detected rather than SP. The MES technique in (5) may not yield an accurate ToA (range) estimate in the presence of MPCs prior to the strongest one. In order to improve the accuracy of range estimation, (Falsi et al., 2006) proposes to consider the largest $\mathrm{N}$ correlation peaks, and to select the time delay corresponding to the peak with the smallest time index. In (Falsi et al., 2006), the authors also propose two improvements for this technique in non-resolvable channels.

- Ranging with Two-Step ToA Estimators

It is of significant importance to perform accurate range estimation without employing high sampling rates in UWB ranging systems. In order to have low-power and low-complexity implementations, symbol-rate or frame-rate samples should be considered (Gezici et al., 2005), which can, however, increase the time to perform ToA estimation considerably. One technique to perform reasonably accurate range estimation based on low rate samples is to employ two-step ToA estimators (Gezici et al., 2008). In order to perform ToA estimation in short time intervals, the first step of the two-step ToA estimator in ( Gezici et al., 2008) performs a coarse timing estimate using energy detection (similarly, the dirty template approach can be used). Then, the second step refines the ToA estimate based on a statistical change detection algorithm (Guvenc et al., 2008).

- Ranging with Dirty Templates

Another low complexity ToA estimator is the dirty-template technique introduced in (Yaung \& Giannakis, 2004), (Yaung \& Giannakis, 2005), which operates on symbol rate samples. The main idea behind this technique is to use the received signal itself as a dirty template and then to perform cross-correlations with the symbol-length portions of the received signal. The main advantage of the dirty-template technique is its low complexity, however, the estimates obtained by this technique can have an ambiguity equal to the extent of the noise-only region between consecutive symbols. 


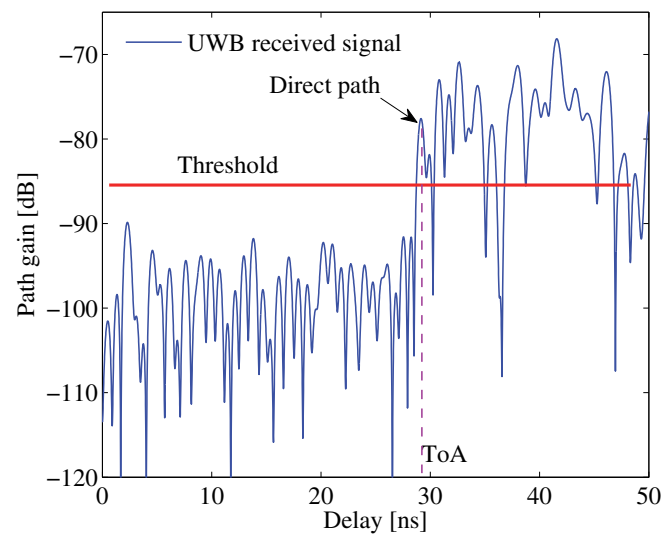

Fig. 5. Threshold-based ToA estimation on received UWB signal

- Threshold-based Ranging

Threshold based ranging algorithms compare the received signal samples against a threshold in order to identify the first arriving MPC (Dardari \& Win, 2006). In (Scholtz \& Lee, 2002), a simple threshold based algorithm is introduced, which compares absolute values of the direct samples of the received signal (or, samples of the correlator/energy detector output) against a threshold. The ToA of the signal is determined by the index of the first sample that exceeds the threshold. Threshold-based ToA estimation algorithm, in general, is illustrated on Fig. 5. Since the signal energy and the channel impulse response are unknown, the threshold can be determined based on the noise floor. In next section, a detailed survey on threshold setting is presented.

\subsection{Survey on threshold-based ranging techniques}

In previous sections, it was explained that the signal peak detection for the estimation of the $n_{0}$, discussed in (Haneda et al., 2009), (Sahinoglu et al., 2003), is not reliable in multipath environments even in LoS scenario due to multipath interference to the first arriving signal. The simplest approach then is comparing the received samples to a presumed threshold level. In the threshold-based ranging systems after acquiring the received signal sample energy from (3), the decision is made on the first threshold-exceeding sample $n_{\mathrm{D}}$. The corresponding delay can be obtained as

$$
\begin{aligned}
\tau_{\mathrm{D}} & =n_{\mathrm{D}} T, \\
n_{\mathrm{D}} & =\arg \min _{n}(z(n)>\xi),
\end{aligned}
$$

where $\xi$ is a presumed threshold. Threshold-based ToA estimation is specifically attractive due to its low complexity and computational burden which are crucial for many low cost battery powered devices, e.g. wireless sensor networks and radio frequency identification (RFID). These search algorithms compare individual signal samples with a certain threshold in order identify the first arriving signal and obtain the range information (Dardari \& Win, 2006). The important challenge in the threshold-based approach is how to pick up a threshold level to have least ToA estimation error which is equivalent to the minimum ranging error. In next section, a review on the existed threshold-setting techniques is given. 


\subsubsection{Threshold setting techniques}

Few threshold selection methods have been proposed in the literature, in fact in most researches a known optimum threshold, usually derived by trial and error, is presumed. In (Dardari \& Win, 2006) it was observed that the performance of the ToA estimation depends on the threshold value but no comment was delivered on how to set it. It is reported that the threshold-based estimator is more convenient in high signal-to-noise ratio (SNR). Moreover the sensitivity of the system to the threshold value was discussed without proposing how to set it. It was argued that the threshold level can be selected based on the noise level or signal peak (Dizdarevic \& Witrisal, 2007; Haneda et al., 2009). In (Guvenc \& Sahinoglu, 2005), two different threshold based ToA estimation algorithms are proposed. The thresholds of those algorithms can be set based on the noise level, or, using a normalized threshold as in (Guvenc \& Sahinoglu, 2005). In either case, certain statistics of the UWB channels should be used to select parameters for the search space and stopping rules accurately. The problem in those approaches is that the derivation of SNR without any knowledge of the ToA in practice is a challenge itself. The threshold has been selected as a normalized value between maximum and minimum energy samples to improve the performance in (Guvenc \& Sahinoglu, 2005), although the dependency to the SNR and signal level is preserved. In (Guvenc \& Sahinoglu, 2005) the Kurtosis of the signal samples is used as a metric for threshold selection. Unlike the SNR of the received signal, Kurtosis captures both the statistics of individual channel realizations, and the relative energy of the signal to noise. It is shown that using the Kurtosis metric, estimation error can be significantly decreased compared to fixed threshold. In the so far discussed methods the threshold is always assumed a fixed value. The common disadvantage for all of these methods is that they use a presumed threshold value, which is system and environment dependent. In ( Xu \& Law, 2008) and (Dashti et al., 2008) a threshold based on the delay of first arriving signal was proposed. Proposing the delay-based threshold selection technique is postulated on the fact that the first arriving signal power decreases as wireless nodes separate from each other due to the propagation loss. Consequently the threshold for detecting the signal is a function of delay. In ( Xu \& Law, 2008) the threshold value is an exponential function whose parameters are obtained by solving a numerical equation with a knowledge of the noise level and through a further optimization process. The design parameters of the threshold are sensitive to the channel condition and still it is necessary to predetermine the noise level. The common problem of the discussed threshold setting methods is that none of them was optimized based on the design parameters such as the ranging error. The problem of threshold-setting is experimentally addressed in next section.

\section{Ranging based on measured propagation channels}

To examine the performance of ToA-based ranging in the real scenario a measurement campaign was conducted in an office room. The threshold-based ranging algorithm was applied to get the range of the measured data $d_{\mathrm{m}}$. Since the real separation of the transmitter and receiver $d_{0}$ is well known from the coordinates of the transmitter and receiver nodes, the experimental ranging error is obtained as

$$
e_{\mathrm{m}}=d_{\mathrm{m}}-d_{0}
$$

The ranging error is used as criteria to evaluate the ranging algorithms. The details of the measurement and ranging results are given in next sections. 


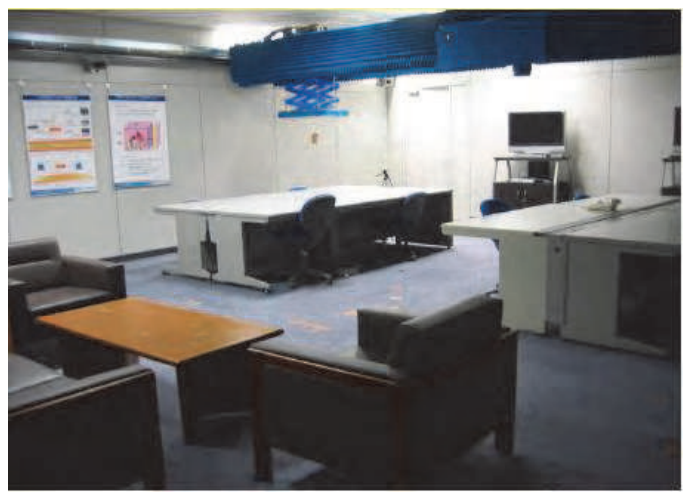

Fig. 6. The view of the measurement environment

\subsection{Experiment scenario and channel measurement results}

The database is obtained in the typical office room in National Institute of Information and Communications Technology (NICT), Japan. The view and layout of the measurement environment are shown in Fig. 6 and 7. The description of the experiment is as follows. The transmitter antenna could be positioned at almost any place in the room by the aid of a large spatial precision scanner covering the whole areas of the room, whereas the receiver antenna was fixed at the corner of the room. The transmitter to receiver distance varied from 0.6 to $9.3 \mathrm{~m}$. In total, 4200 spatial samples of transfer function were measured on the transmitter side by a vector network analyzer (VNA). The maximum detectable ToA was 200 ns, sufficient to capture dominant propagation paths. LoS was assured in most Tx positions except for two arrays where LOS was obstructed due to television displays. The whole measurement took about 6 hours, but there was no moving object during the measurement so that the time-invariance of the angular-delay channel characteristics was ensured. Phase drift of the VNA was carefully compensated during the measurement by performing an internal calibration procedure of the VNA once in two hours. Details of the measurement specifications are summarized in Table 1, for more details please refer to (Dashti et al., 2008). Since the measurement data and all the analysis in this study were done in allocated sub-bands in IEEE 802.15.4a standard, the specifications of this standard are briefly described. The IEEE802.15.4a standard defines several frequency bands for the realization of piconets as summarized in Table 39i-UWB PHY band allocation in (IEEE Std, 2007). The band plan defines three band groups: sub-gigahertz, low, and high bands. The frequency band of interests is selected from the band plan of IEEE802.15.4a-2007 standard. The band plan defines low and high bands spanning from 3.1 to $4.8 \mathrm{GHz}$ and from 6.2 to 10.3 $\mathrm{GHz}$, respectively. Bandwidth of each channel is either 0.5 or more than $1.0 \mathrm{GHz}$. Transfer functions measured within the band of interests are extracted for data processing. We will not consider sub-gigahertz band, since the measurement data do not cover that frequency. In the low and high bands, there are mandatory bands where the ranging operation should be assured most (channels 3 and 9), and others are optional. Bandwidth of each mandatory channel is $499.2 \mathrm{MHz}$ with various center frequencies. There are also optional channels with larger bandwidth (channels 4, 7, 11 and 15) with bandwidth of 1331.2 MHz. Ranging accuracy were assessed in all low-band and high-band UWB channels.

The transfer function is converted to a channel impulse response (CIR) by the inverse Fourier transform. Frequency spectrum of the root-raised cosine pulse is applied as a windowing 


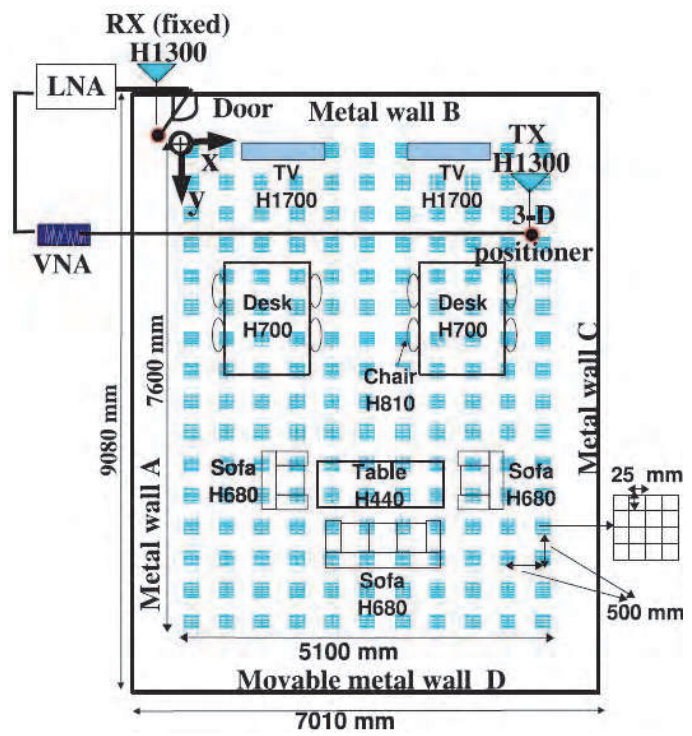

Fig. 7. Layout of the measurement environment

\begin{tabular}{|c|c|}
\hline \multirow{3}{*}{$\begin{array}{l}\text { Frequency band } \\
\text { Band-width (B) }\end{array}$} & $3.1-10.6 \mathrm{GHz}$ \\
\hline & $500 \mathrm{MHz}$ \\
\hline & Vector network analyzer \\
\hline Measurement equipment & $\begin{array}{l}\text { Room-wide spatial scanner } \\
\text { Low-noise amplifier ( } 30 \mathrm{~dB})\end{array}$ \\
\hline No. of frequency sweeps & 1501 \\
\hline Antenna & UWB monopole \\
\hline Transmitted power & $-17 \mathrm{dBm}$ \\
\hline Coverage area dimensions & $5.1 \times 7.6 \mathrm{~m}^{2}$ \\
\hline Wireless nodes range & $0.6 \mathrm{~m}$ to $9.3 \mathrm{~m}$ \\
\hline Wireless nodes height & $1.3 \mathrm{~m}$ above the floor \\
\hline
\end{tabular}

Table 1. Experiment parameters

function. The root-raised cosine pulse is denoted in the time domain as

$$
r(t)=\frac{4 \beta}{\pi \sqrt{T_{\mathrm{p}}}} \frac{\cos \left(\frac{(1+\beta) \pi t}{T_{\mathrm{p}}}\right)+\frac{T_{\mathrm{p}}}{4 \beta t} \sin \left(\frac{(1-\beta) \pi t}{T_{\mathrm{p}}}\right)}{1-\left(\frac{4 \beta t}{T_{\mathrm{p}}}\right)^{2}}
$$

where $\beta$ and $T_{\mathrm{p}}$ is a roll-off factor and pulse length specified in the standard (see (Molisch et al, 2004), pp.82-83). CIR is calculated for all the Tx locations.

Power of the direct and strongest paths is shown in Fig. 8 against Tx-Rx distances. Results from channels 2 and 4, which are in the low band, and 11, which is in the high band, are shown. The figures revealed the following findings. Channels with wider bandwidth show less gain variation of the direct and strongest paths. Comparison of results from channels 2 and 4 revealed that the variation of path gain values is less in channel 4 . The two channels have 

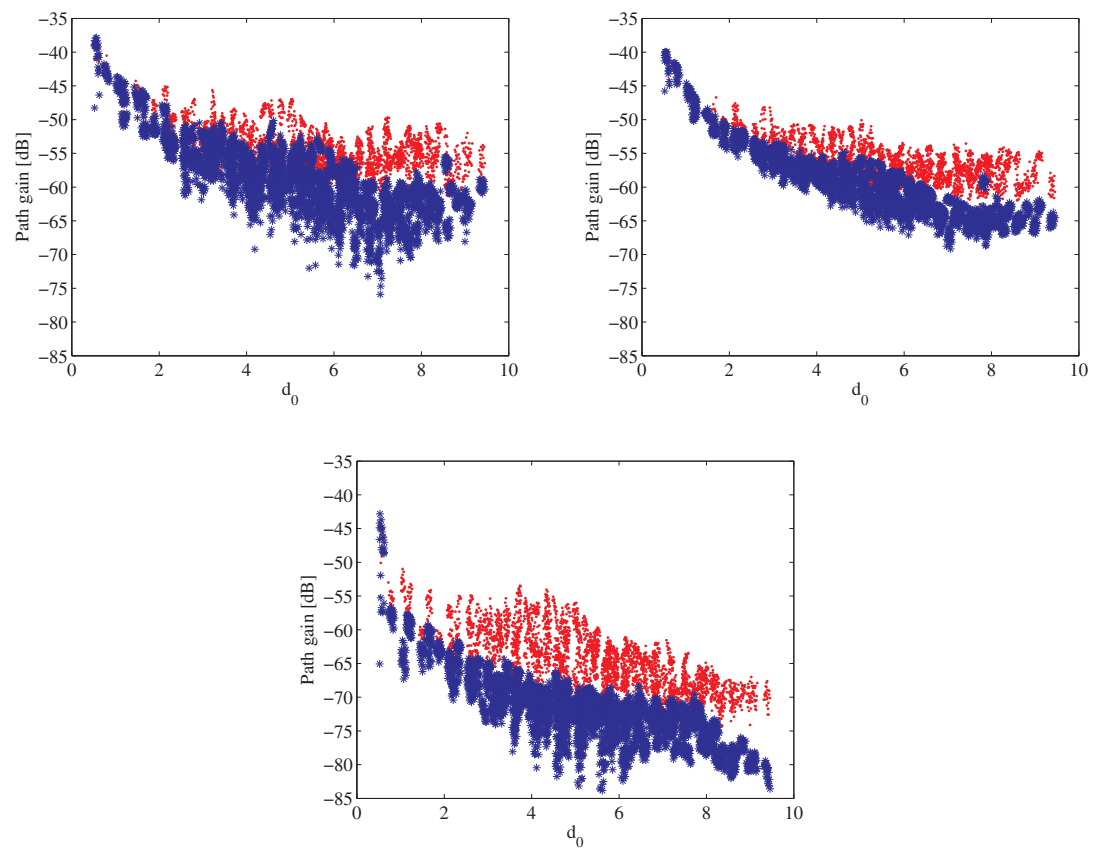

Fig. 8. distribution of measured direct path gain (blue stars) and strongest path (red dots) in channels (a) 2 (b) 4 (c) 11

the same center frequency, but channel 4 has about three times larger bandwidth than channel 2. The narrower bandwidth leads to poorer delay resolution, which causes the fluctuation of power in direct and strongest paths due to the fading with non-resolvable signal components around the paths. As a result, the gain of the first and strongest paths is slightly higher in those channels. The same trend was observed in other channels with the same center frequency and different bandwidths, such as channels 5 and 7, 9 and 11, and 13 and 15. This is the same observation as reported in the work of Alsindi et al. (Alsindi et al., 2007).

Difference of the path gain between the high and low bands are 5 to $15 \mathrm{~dB}$. The path gain in the high band was smaller value than the low band as expected. The largest and smallest gain was observed in channels 1 and 11, respectively. The level of path gain is almost the same in the low band, while $5 \mathrm{~dB}$ gain difference was observed within the high band. Channels 5 and 11 showed the largest and smallest gain in the high band, respectively. The channel with the highest frequency did not show the smallest path gain, probably because of the frequency characteristics of antenna gain.

Fig. 9 shows the example of a measured received signal. It depicted that due to the effect of multipath interference the strongest path is not necessarily the direct path even under the LoS condition. Multipath interference leads to fading and causes the strongest path spread over the delay axis. In ranging analysis, direct path should be detected rather than strongest path. In this example the ToA of direct path is estimated wrongly from expected ToA. The ranging error is modeled in (Dashti et al., 2010). 


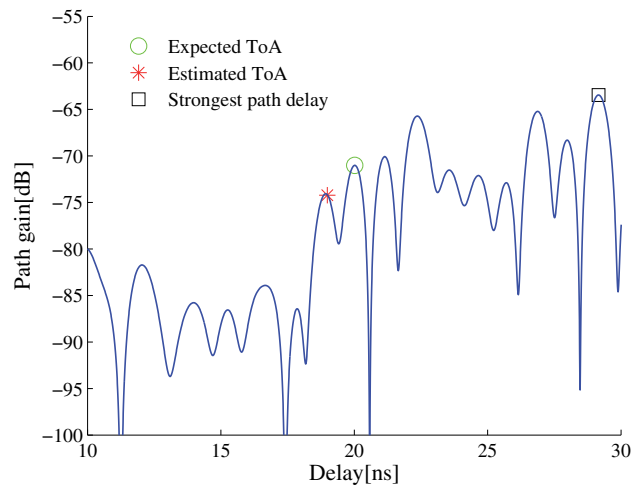

Fig. 9. An example of received signal where the strongest path is in a delay from LoS as a result of destructive multipath interference (Dashti et al., 2010).

\subsection{Ranging with fixed threshold value}

The fixed threshold value can be optimized based on noise level or peak signal level. Two threshold-based methods are introduced to detect the signal component corresponding to the first path: the leading edge detection, which set the threshold based on noise level, and the search back method, which the detection threshold level is given by the power of strongest path (SP). Coherent detection is assumed in both ranging methods. Schematic representation of these two methods is shown in Fig. 10.

\subsubsection{Search-back method}

Search-back method utilizes the strongest path of CIRs to detect the direct path. It has been commonly reported that the first path is not always the strongest path, particularly in NLoS scenarios due to LoS blockage. As it was discussed earlier, this could happen even in LoS situations due to multipath propagation. Specially, power of delayed paths could be greater than the first path because of overlapping multipaths arriving at the same delay time. In other cases, the first path suffers from destructive fading due to surrounding non-resolvable multipaths. The search back method first finds the strongest path, and then looks for a peak arriving before the strongest path which has greater power than a detection threshold level. We proposed an iterative search-back algorithm to calculate the noise floor (NF) to be used in the detection of first path. In the first iteration, the algorithm detects the strongest path, and then calculates the noise floor by averaging over the interval $\left[0,\left(t_{\mathrm{sp}}-t_{\mathrm{c}}\right)\right]$, where $t_{\mathrm{c}}$ is delay resolution. The interval is $t_{\mathrm{c}}$ less than the SP delay to exclude the effect of SP signal. To remove the effect of side lobes, $t_{\mathrm{c}}$ was chosen $1 \mathrm{~ns}$. In next iterations this process is repeated for new time interval $\left[0,\left(t_{\mathrm{i}}-t_{\mathrm{c}}\right)\right]$, and it will continue to find the new peak value and the new NF. Here $t_{\mathrm{i}}$ is the time delay of the peak detected in the $i-$ th iteration. The algorithm will be continue until finding the first peak higher than the NF by predefined search-back threshold value, $\gamma_{S}$, which is dependent on system bandwidth. Fig. 11 shows the flowchart of the proposed iterative algorithm. $P_{\mathrm{i}}$ and $N F_{\mathrm{i}}$ in the flowchart are peak value and NF in the $i-$ th iteration. Obviously the value of NF is erroneous in the first iteration but it will give the real NF and first detected path after enough iterations. $\gamma_{S}$ level which the algorithm used for detecting of first path is chosen different for each subband. To obtain the optimum $\gamma_{S}$ which gives lowest error, we calculated the ranging error using several $\gamma_{S}$, such as $5,10,15$ and $20 \mathrm{~dB}$. Concerning 


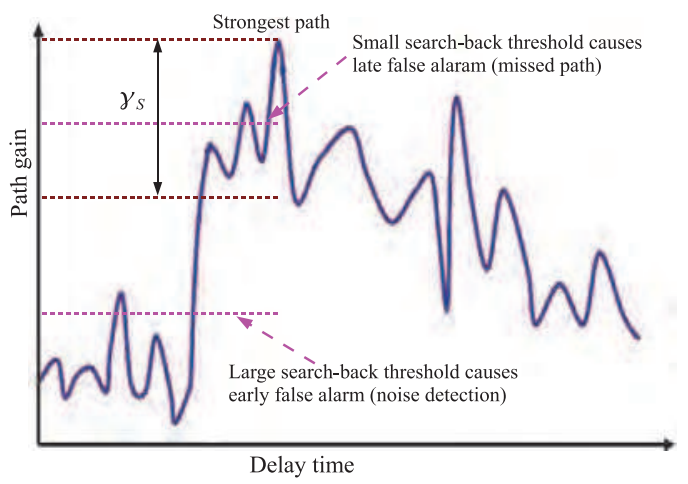

(a)

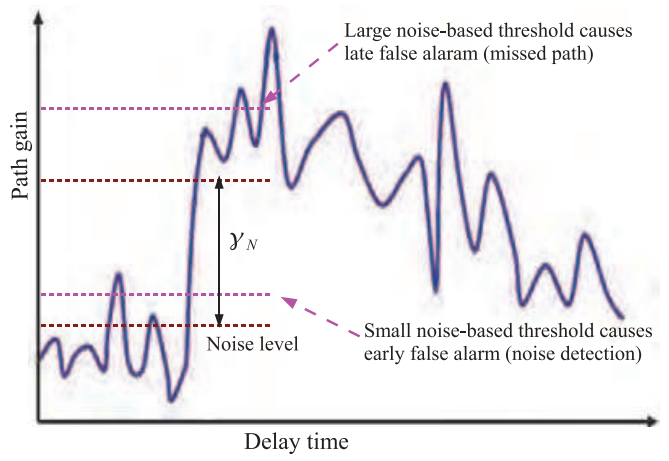

(b)

Fig. 10. (a) Search-back detection method vs. (b) leading edge detection method

the difference of SP signal level in different sub bands, these optimum $\gamma_{S}$ values are different for channels with different bandwidth. For instance, $\gamma_{S}$ was chosen $15 \mathrm{~dB}$ for Channel 4 . Same analysis was done for the other subbands, however we hesitate to show the ranging results of all of them for the sake of conciseness. For higher BW the algorithm search for the first peak above $15 \mathrm{~dB}$ from NF, $\gamma_{S}$ is chosen $10 \mathrm{~dB}$ for channels with lower BW. It is observed that the NF decreases for higher bands and also decrease by increasing the bandwidth. The peak value decreases in higher bands and also decreases by increasing the bandwidth. Since path loss increases as the frequency increases. This algorithm has the advantage of obtaining the result after a few number of iterations for the far points. Also for the close points (Tx and Rx close together) in the lower frequency bands, the averaging over longer intervals in the first iteration seems to be reliable by using this algorithm. For instance for an arbitrary position in the room in channel 3, by applying the mentioned iterative algorithm, after only 2 iterations, we could detect the correct first path. The ranging error for this position is $0.2 \mathrm{~m}$, which is a relatively small error while the real distance between $\mathrm{Tx}$ and $\mathrm{Rx}$ is $4.6 \mathrm{~m}$. However the required ranging accuracy depends on the application. The calculated NF for this position 


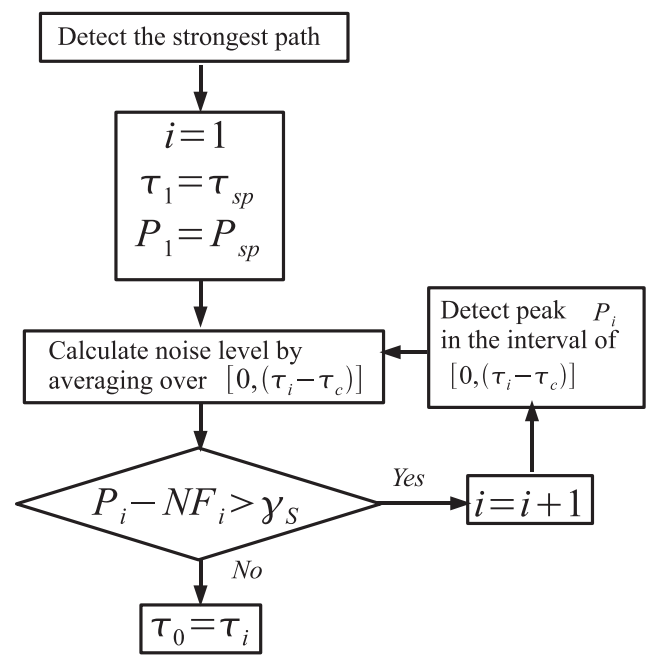

Fig. 11. Flowchart of search-back algorithm

is $-72 \mathrm{~dB}$. The power level of first path is $14.2 \mathrm{~dB}$ more than the calculated NF. Evaluation of ranging accuracy were assessed in all channels. The ranging result shows the algorithm works well for almost all of the positions, however ranging errors are observed in some cases. We categorized the ranging errors to two main categories, relatively small positive/minus errors and large positive/minus errors. When the peak of channel response gets a little shifted from the expected ToA to shorter/longer ToA, resulting small errors in ToA estimations. In some far positions from the Tx antenna large ranging errors are observed. These large errors may be produced by the occurrences of undetected path conditions, or false estimation of NF by proposed algorithm. For instance in an arbitrary position where large minus error happened, the calculated NF for that point is $-104 \mathrm{~dB}$, and the first detected path level is $14 \mathrm{~dB}$ higher than this NF, however this peak is not the real first arrival path, so causes relatively large minus ranging error. In the proposed first path detection, the detecting of first peak started from SP , going to the origin, and it continues till finding the first peak higher than calculated NF by $\gamma_{S}$ value. This algorithm has the advantage of detecting the peak after a few iteration numbers in many cases. However for some cases the algorithm cannot detect the first path, and SP is detected as first path. Detection algorithm started from origin and going to SP may eliminate the error of such these cases. In following leading edge algorithm is described.

\subsubsection{Leading edge method}

In leading edge method, the fixed threshold value can be optimized based on noise level. We refer this method as noise level based threshold. Leading edge detection is the most primitive method to detect the first path. The device monitors a time series of correlator outputs in a coherent detector. Provided that the power monitor, like a received signal strength indicator in a general receiver, knows the noise level of the receiver in advance, it can detect the first path when a signal level exceeds a certain level. The first output sample exceeding noise level by a predefined threshold value will be detected as ToA, i.e. ToA is the delay time of the 


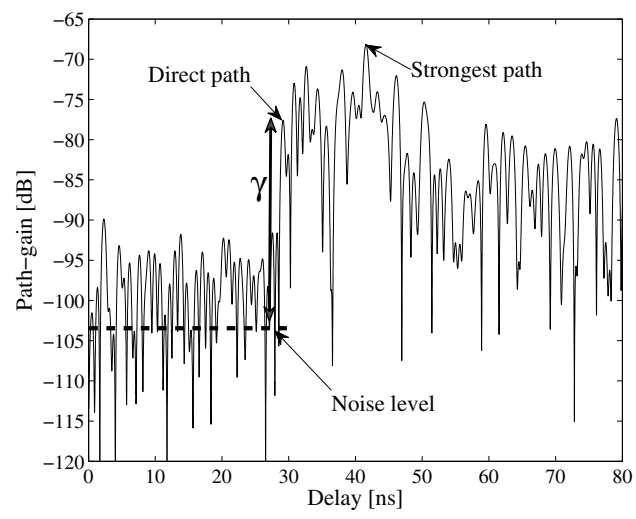

Fig. 12. Noise level based threshold for ToA estimation

earliest received sample that fulfills the condition of:

$$
\begin{aligned}
\tau_{\mathrm{D}} & =n_{\mathrm{D}} T \\
n_{\mathrm{D}} & =\underset{n}{\operatorname{argmin}}\left(z[n]>l_{\mathrm{N}}+\gamma\right)
\end{aligned}
$$

where $\gamma$ is the presumed fixed threshold value and $l_{N}$ is the noise level. $\gamma$ can be optimized for individual UWB subbands in order to have the minimal ranging errors. The principle of noise level based ToA estimation algorithms is summarized in Figure 12. However, there are two cases the method fails to detect the first path: miss and noise detection. The miss detection (late false alarm) occurs if the level of the detection threshold is greater than the power of the fist path, while the noise detection refers to the case where a noise peak is wrongly detected as the first path. The noise detection is regarded as a early false alarm.

The Fig. 13 shows the superior performance of leading edge against search-back method for channel 3. The ranging results in all channels revealed that the leading edge detection always outperforms the search back method. This is because the search back method uses strongest path. As reported in the channel modeling result, strongest paths fluctuate in power, resulting in larger fluctuation of the level difference between the first and strongest paths. Therefore, the search back method needs to increase the search back level in order to capture the first path perfectly. The larger search back level, however, results in increasing probability of noise detection, resulting in the degradation of the mean detection probability. On the other hand, the leading edge detection suffers from the power fluctuation less. According to the channel modeling result, smaller power fluctuation was observed in channels with wider bandwidth. In such channels, the first path detection probability of the search back method is comparable with that of the leading edge method. The search back method achieves perfect detection probability on the diagonal line of the room, but miss and noise detection starts to occur once the Tx location is getting off from the diagonal line. This means that the performance is largely dependent on spatial multipath characteristics. While it was not found in the leading edge detection because of its robustness to the varying multipath structure. The miss detection is most visible in near-wall Tx locations. It is generally seen that in leading edge method, smaller path gain leads to lower threshold values in order to capture first paths correctly. Hence the threshold value indicates larger values when it is optimized in the limited areas to rule out 


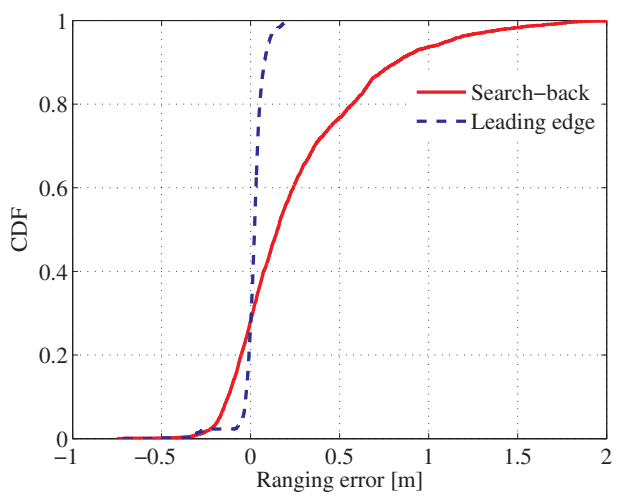

Fig. 13. Comparison of leading edge and search-back methods

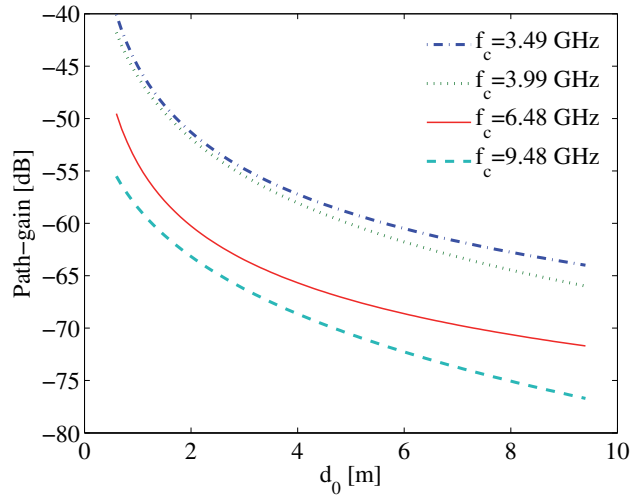

Fig. 14. Direct path-gain in different subbands with different center frequencies

Tx locations with low signal levels. The same trend is observed in the search back level, but the fluctuation of the value is small over different center frequencies and bandwidths In the leading edge method named noise level based threshold approach, noise level can be assumed initially as a fixed value or can be calculated based on initial part of the signal. We categorized noise level based threshold ToA estimation concerning presumption or estimation of noise level. In following section more description is given.

- Presumed noise level A prior knowledge about the noise can be assumed to set the $l_{\mathrm{N}}$ as a single value, i.e. in equation $(10), l_{\mathrm{N}}$ is presumed single noise level. We assumed thermal noise level given by $l_{\mathrm{N}}=k_{\mathrm{B}} T_{\mathrm{k}} B$ where $k_{\mathrm{B}}$ is the Boltzmann constant, $B$ is the system bandwidth and $T_{\mathrm{k}}$ is the absolute temperature in kelvin.

Fig. 14 shows the best fit for the measured FAP path gain as a function of Tx-Rx distances for different channels. It is observed that the FAP path gain decreases in higher subbands since the path loss increases, Hence $\gamma$ in equation (10) was optimized for each channel individually in order to have minimal ranging errors. Fig. 15 shows the optimum value of threshold for all different channels. $\gamma_{\text {opt }}$ varies from $30 \mathrm{~dB}$ for channel No.1 with lowest 


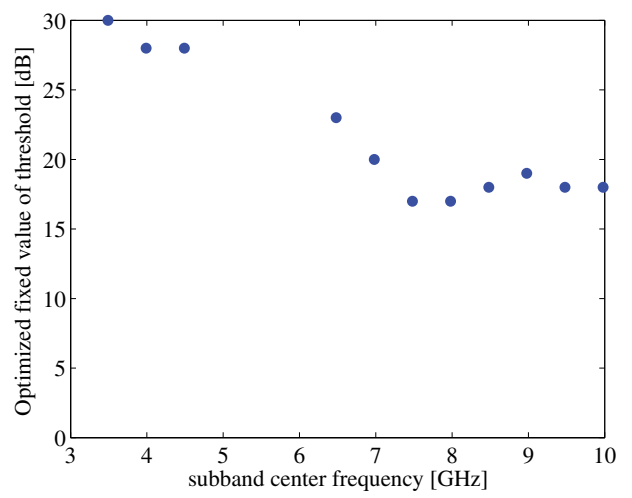

Fig. 15. Optimized fixed value of threshold for different subbands with different center frequencies

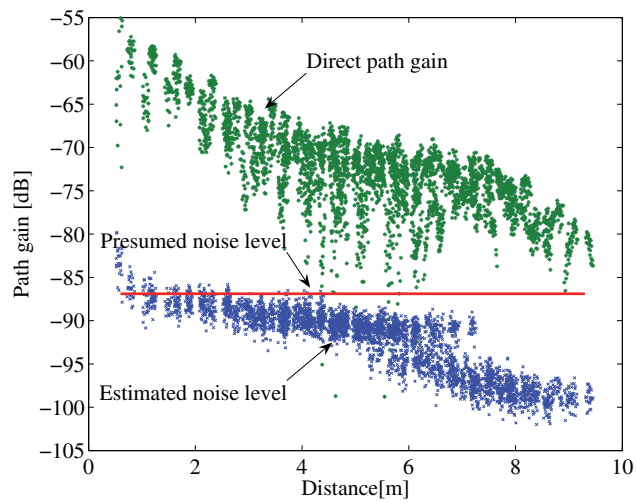

Fig. 16. Measured direct path gain against presumed and estimated noise level

center frequency, $f_{c}=3.49 \mathrm{GHz}$, to $15 \mathrm{~dB}$ for channel No.15 with highest center frequency, $f_{c}=9.98 \mathrm{GHz}$, Fig. 15 shows direct path gain compared with presumed fixed $l_{\mathrm{N}}$. The direct path gain decreases with longer Tx-Rx distance while noise level is a single value, therefore the differences of direct path gain and noise level are not a single value for all Tx-Rx distances. As Fig. 16 shows, the difference between direct path gain and $l_{\mathrm{N}}$, vary in a wide range. Due to this wide variation, presetting a single value for $\gamma$, which gives minimal ranging errors for all possible Tx-Rx distances, is a challenge.

- Estimated noise level In this approach instead of presuming a single noise level, we estimate the noise level based on the initial part of the received signal, i.e. in equation $(10), l_{\mathrm{N}}$ is not a single value but it is calculated for each channel realization. Fig. 15 shows direct path gain compared with estimated $l_{\mathrm{N}}$ for different Tx-Rx distances. In (Dashti et al., 2008) the variance of ranging error of estimated noise level approach with those obtained by presuming the $l_{\mathrm{N}}$ are compared. It was shown that by estimating $l_{\mathrm{N}}$, variance of ranging error dramatically decreases in all channels, However still the algorithm fails in some cases. Setting a fixed threshold value is not reliable due to variation of direct 
path gain on different Tx-Rx distances. Since direct path gain decreases with longer Tx-Rx distance, threshold value also can be set to decrease with Tx-Rx distance. We proposed a delay-dependent threshold selection method in next section.

\subsection{Ranging with delay-dependent threshold setting}

In previous section two fixed threshold based methods (leading edge vs. search-back) are introduced and the ranging performance of them are compared. The performance degradation in the search back method is due to the gain fluctuation of the first and strongest paths, which is most remarkable in the high band. The selection of the optimum threshold level for these two ranging methods still remains an important issue.

As it was described in previous section, we introduce a technique to set the threshold as a function of Tx-Rx distance instead of a fixed value as in conventional noise level based threshold methods. In this method, we preset a delay-dependent threshold function $\xi(n)$. The received samples are then compared to the respective threshold values, $\xi(n)$. The arrival time of the first sample crossing the respective threshold value within time interval $\left[0, t_{\mathrm{SP}}\right]$ is estimated as ToA, where $t_{\mathrm{SP}}$ is the delay time of the SP. Fig. 17 (a) shows the basic of the proposed method. In this method estimation or assumption of noise level is not needed. As described, algorithm searches for a first received sample crossing its respective threshold. In some cases there is no peak located in the detected sample, $n_{\mathrm{D}}$ th sample, as shown in Fig. 17 (b), due to resolution of system and algorithm. The algorithm then search for a nearest peak value in the interval of $\left[n_{\mathrm{D}} T-t_{c}, n_{\mathrm{D}} T+t_{c}\right]$, where $t_{c}$ is set according to the resolution of system.

As a reliable delay-dependent threshold the standard path-gain model is employed, which is to predict the expectation of $E_{n_{0}}$ at any indoor position, according to the IEEE802.15.4a standard channel model (Molisch et al, 2004). This model is generic and widely used for the indoor UWB channel modeling applications. In following IEEE802.15.4a standard path gain model is briefly explained. The parameters of the model are also extracted by fitting measurement data to the described path gain model.

In the IEEE802.15.4a standard, path gain in a UWB channel is defined as:

$$
G(f, d)=G(f) G(d)
$$

Path gain is a function of the distance and frequency. In this model, it is assumed that the distance and frequency dependent effects are spreadable. The separation reduces the complex two-dimensional path gain modeling to one-dimensional problem. The frequency dependency of the channel path gain is modeled as:

$$
G(f) \propto \sqrt{f^{-k}}
$$

In IEEE802.15.4a model the distance dependence of the path gain is described by the conventional power law for simplicity as:

$$
G(d)=G_{\mathrm{R}} \times\left(\frac{d}{d_{\mathrm{R}}}\right)^{q}
$$

Combining (11), (12) and (13) yields the following equation in $\mathrm{dB}$ for total path gain.

$$
G(d)=G_{\mathrm{R}}-20 k \log \left(\frac{f}{f_{\mathrm{R}}}\right)-10 q \log \left(\frac{d}{d_{\mathrm{R}}}\right)
$$




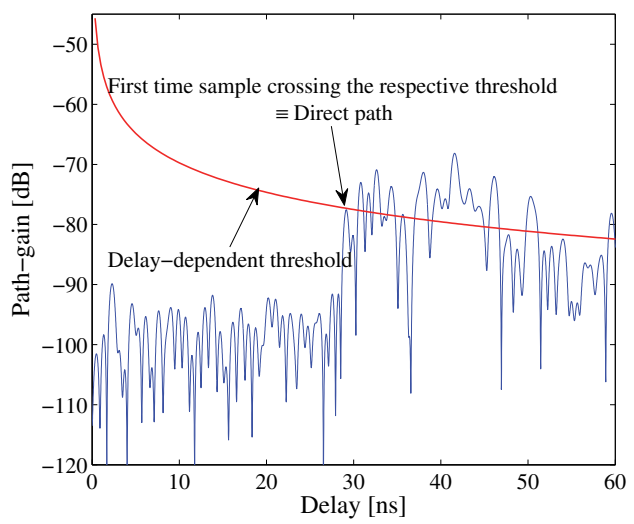

(a)

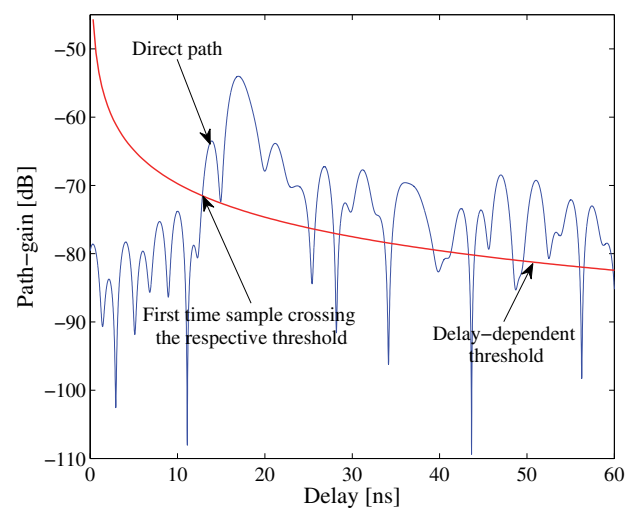

(b)

Fig. 17. An example of Delay-dependent threshold against a measured channel impulse response a) a peak located in $n_{\mathrm{D}}$ sample b) $n_{\mathrm{D}}$ sample is not a peak hence algorithm search for nearest peak in the interval of $\left[n_{\mathrm{D}} T-t_{c}, n_{\mathrm{D}} T+t_{c}\right]$

which in essence states that the path-gain is influenced by attenuation due to the frequency $f$ and the transmitter to receiver separation $d$. The decaying exponent due the frequency and the distance are expressed as $k$ and $q$, respectively while $G_{R}, f_{R}$ and $d_{R}$ are the reference path-gain, frequency and distance respectively (Molisch et al, 2004).

Fig. 18 shows the distribution of measured path gain within the scanned area in the room. $\mathrm{X}$-axix and $\mathrm{Y}$-axix represent the coordinate of the transmitter in $\mathrm{X}-\mathrm{Y}$ plane in the area covered. Measured direct path gain distribution for lowest and highest subbands, which are channel 2 and 14 respectively, are shown in the Fig. 18 (a) and (b) . Figure depicts the dependency of the path gain to the distance and frequency. The parameters of the model were extracted by fitting measurement data to the described path loss model. Following procedure was performed for determination of model parameters similar to method presented in (Haneda et al., 2007): 

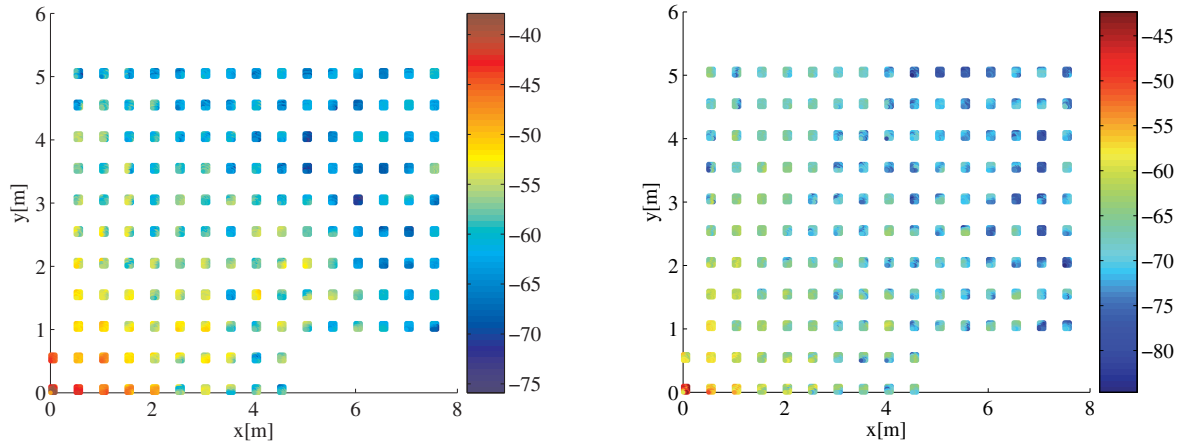

Fig. 18. distribution of measured direct path gain within the scanned area in the room in channels (a) 2 (b) 14
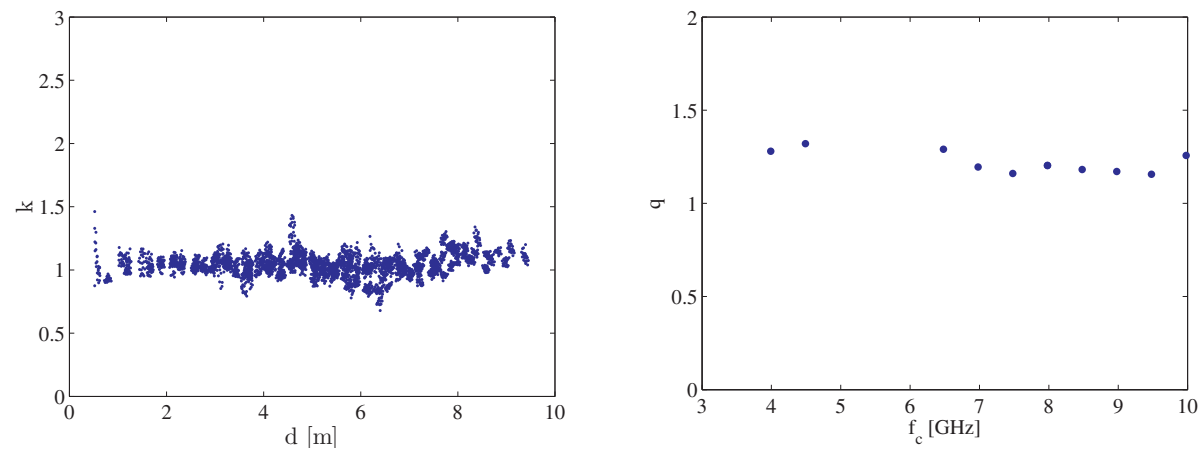

Fig. 19. Path loss model parameters (a) Dependency of $k$ to Rx-Tx distance (b) Dependency of $q$ to frequency

- Frequency decaying factor determination: Frequency decaying factor, $k$, was derived using equation (12). To observe the variation of $k$ on the Tx-Rx distance, derivation was done for all the possible Tx-Rx distances. It was assured that the frequency decaying factor is almost constant for all possible distances. The variation range was between 0.96 to 1.22 and the mean value is 1.12. Dependency of $k$ to Rx-Tx distance is shown in Fig. 19(a).

- Distance decaying factor determination: the distance decaying factor, $q$, was derived using (13). To observe the variation of the distance decaying factor, derivation was done for all possible frequency samples. It was assured that the variation of $n$ is negligible for different frequency samples. The variation of $q$ was between 1.15 to 1.32 . The mean value of all samples, 1.22, could be represented the distance decaying factor. Dependency of $q$ to frequency is shown in Fig. 19(b).

- Initial path gain determination: initial path loss value, $G_{R}$, was calculated using (14) by replacing the obtained frequency and distance decaying factor from the above two steps. Obtained $G_{R}$ from our measured data was $39.07[\mathrm{~dB}]$. 
The specific values for these parameters for the indoor LoS scenario are reported as $f_{R}=5$ $\mathrm{GHz}, d_{\mathrm{R}}=1 \mathrm{~m}, \mathrm{G}_{\mathrm{R}}=-35.4 \mathrm{~dB}, k=0.03$ and $q=1.6$ for the indoor office and $G_{\mathrm{R}}=-43.9 \mathrm{~dB}, k$ $=1.12$ and $q=1.8$ for the residential environment (Molisch et al, 2004). Following the same approach, corresponding parameters for the measured values were derived as $G_{R}=-39 \mathrm{~dB}, k=$ 1.12 and $q=1.22$. These parameters are slightly different from those proposed by the standard model due to specific environment. Good fit, typical for all subchannels, is observed which indicates the appropriateness of the model to be used for the threshold setting.

The standard path-gain formula was applied as the proposed delay-dependent threshold to get the range of the measured data $d_{\mathrm{m}}$. Standard deviation of the ranging error $\sigma_{e}$ obtained from the path-gain threshold and the best fixed threshold are presented in (Dashti et al., 2009). It is observed that the path-gain threshold gives a lower ranging error in all subchannels with a stable performance over all frequency bands. The performance of the fixed threshold ranging however is frequency dependent due to different path-loss and interference (Dashti et al., 2008).

\subsection{Effect of center frequency and band width}

An important finding from Fig. 8 is that given the wide dynamic range of signal levels over varying distance, it is hard to find one optimum threshold which achieves the perfect direct path detection everywhere in one office room. The inherent problem here is that the limited transmit power hinders the signals from reaching more than several meters away.

Another finding is that channel 4 is able to provide reliable ranging in almost all the locations of the room. The result from channel 7 indicated that the noise detection is the main source of error in many Tx locations. In wall-side Tx locations, however, the miss detection becomes a dominant source of error. The miss detection is attributed to the weak direct paths close to the noise level, making its detection difficult. The results of channel 11, which showed the smallest path gain among the channels, is dominated both by the noise and miss detection. In that channel, even the strongest paths are as weak as, or weaker than the noise level. Systems operated in the high band often faces this issue. It is therefore very essential to introduce a technique to improve the signal to noise ratio, such as channel averaging functionality for noise reduction and beam forming for increased signal level, in the receiver. Accurate ranging in the low band is promising even under the transmit power restrictions, while the use of high band necessitates a fundamental countermeasure against the low signal level at the receiver. It turned out that the gain of direct and strongest paths quickly decreases with increasing frequency. The restriction of the transmit spectral density further limits the service coverage. Still, ranging in the low band reveals promising performance, while accurate ranging can only be performed in a very limited areas in the high band. For example the ranging method in the highest frequency band allows accurate ranging only within $1 \mathrm{~m}$ range relative to the device. This fact implies that accurate ranging in NLoS scenario is even less promising due to excess path loss due to whatever path obstruction. It is important to note that the most influential factor in the accurate ranging in NLoS scenarios would be the limited transmit power, rather than the LoS blockage and multipath propagation.

It is also found that the detection probability has obvious dependency on the bandwidth. There are four combinations of bands with the same center frequencies and different bandwidths. It was found that channels with wider bandwidths give rise to lower detection probability. The trend becomes remarkable as the frequency increases. This is a natural consequence of the observation in the channel modeling that the wider bandwidth gives the lower power of the direct and strongest path, which resulted in increased probability 

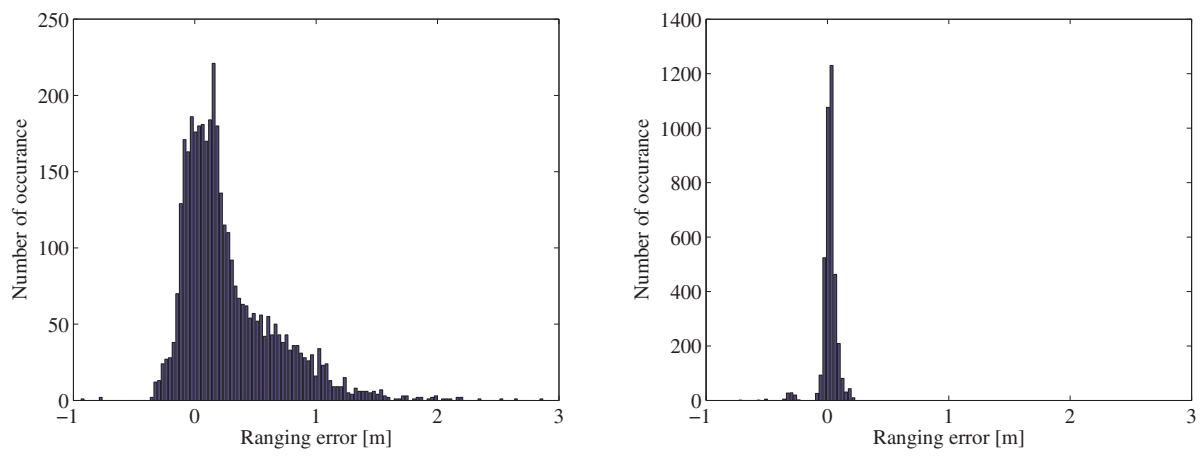

Fig. 20. Histogram of the ranging error (a) channel 5 with bandwidth of $500 \mathrm{MHz}$ (b) channel 7 with bandwidth of $1.0 \mathrm{GHz}$

of noise detection. From the obtained ranging results in different subbands, it is observed that wider bandwidth provides better estimation accuracy of the distance because of the finer delay resolution, as is commonly reported. In Fig. 20 the histogram of ranging errors in channel 5 and 7 with the bandwidth of $500 \mathrm{MHz}$ and $1.0 \mathrm{GHz}$ is shown. It can be concluded that wider bandwidth always gives better detection and estimation accuracy of the direct paths in the low band, while the superiority of the wider bandwidth is becoming less visible as the center frequency goes higher. Use of wider bandwidth does not always provide better ranging performance, particularly in the high band. In contrast to the well-known observation that wider system bandwidth gives rise to better accuracy of range estimation, performance of the range detection revealed the opposite trend, particularly in the high band. This is because wider bandwidth leads to lower gain of direct and strongest paths. Systems with wider bandwidth clearly outperforms those with narrower bandwidth in the low band, but that would not be necessarily the case in the high band.

\section{Summary and future trends}

In this chapter the motivations for research on indoor ranging/localization using ultra-wideband systems is described and a literature review is given. UWB time-based ranging and ToA estimation algorithms are reviewed and threshold-based ToA estimation algorithm is provided. A measurement campaign for the indoor ranging is introduced and the obtained results are inspected. A practical method is proposed for setting the threshold value. This method is based on the path-loss of the signal which can be predicted by the standard channel model. The applicability is checked experimentally. The effect of bandwidth on distribution of the ranging error is discussed. There are a few directions that one might take to extend this research:

- A practical threshold setting technique is introduced based on the standard channel model for the indoor environments (Dashti et al., 2011). Proposed threshold setting technique is validated using a set of channel measurement data acquired in a typical office room. More channel measurement should be performed in different indoor environments in order to validate the applicability of the proposed threshold-setting technique in different environments to evaluate the generality of the method. 
- Some practical issues remain unresolved. In particular perfect clock synchronization between transmitter and receiver is assumed. This assumption is unlikely in practice. Solutions to this problem like round-trip measurement have been mentioned, but they need to be implemented and validated in practice. At a deeper level, understanding and quantifying how the synchronization error impacts the accuracy will help in designing a practical system.

- In the system model explained, it is assumed that the transmitter sends out a UWB waveform. It is known that the UWB waveform is distorted during interactions to the wireless channel. For the simplicity of the simulation it is assumed that this distortion is negligible, one might take a more practical received signal to extend this research.

- More practical scenario should be considered, the case that UT antenna pattern is distorted by near objects and the UT orientation is random. Ranging results with the antenna proximity to the human head are presented in (Dashti et al., 2010). It should be noted that the human body is just one of the sources of distortion. Even it is quite possible that the antenna pattern is distorted by the antenna itself and the chassis of UT. Deep understanding of antenna pattern distortion and its effect on ToA estimation can be considered.

- Since this research area is fairly new, there are many different and important ways to contribute to indoor localization technology. There is a need for comprehensive measurements and modeling for indoor localization specific applications. As such the emerging UWB technology promises a solution for combating the indoor multipath condition. As a result the implementation of UWB measurement system and indoor channel modeling for localization is an important area for further research. In addition, analyzing the effect of bandwidth on the ranging error could be accomplished by examining bandwidths in excess of $60 \mathrm{GHz}$. The following can also be conducted as a continuation of the research work, namely, comparing the performance of super resolution algorithms to the UWB system for indoor localization.

\section{References}

Alsindi, N., Alavi, B. \& Pahlavan, K. (2007). Measurement and modeling of ultra wideband TOA- based ranging in indoor multipath environments, In: IEEE Trans. Veh. Tech.

Dardari, D. \& Win. M. (2006). Threshold-based time-of-arrival estimators in UWB dense multipath channels, In: Proc. IEEE Int. Conf. Commun. (ICC), pp. (4723-4728), vol. 10, Istanbul, Turkey

Dashti, M., Ghoraishi, M., Haneda, K., Takizawa, K. \& Takada, J. (2008). Distance dependent threshold ToA estimation, In: Proceedings of IEICE WBS Technical Meeting, WBS2008-53

Dashti, M., Ghoraishi \& Takada, J.(2009). Optimum Threshold for Ranging Based on ToA Estimation Error Analysis, In: 20th Personal, Indoor and Mobile Radio Communications Symposium 2009(PIMRC09)

Dashti, M., Khatun, A., Laitinen,T., Al-Hadi, A.A., Haneda, K., Ghoraishi, M. \& Takada, J. (2010). UWB Ranging with Antenna Proximity to the Human Head, In: APMC 2010

Dashti, M., Ghoraishi, M., Haneda, K., Takizawa, K. \& Takada, J. (2010). Sources of ToA Estimation Error in LoS Scenario, In: ICUWB 
Dashti, M., Ghoraishi, M., Haneda, K., Takizawa, K. \& Takada, J. (2011). Statistical Analysis of Ranging Error and Optimum Threshold for Indoor UWB Positioning, Submitted to: EURASIP

Dizdarevic, V. \& Witrisal, K. (2007). Statistical UWB Range Error Model for the Threshold Leading Edge Detector, In: International Conference on Information, Communications and Signal Processing, ICICS

Ellis, J. \& Rouzet, P. (2004). P802.15.4a Alt PHY selection criteria, In: doc. IEEE802.15-04-0232-16-004a.

Falsi, C., Dardari,D., Mucchi,L. \& Win,M. Z. (2006). Time of arrival estimation for UWB localizers in realistic environments, In: EURASIP J. Appl. Signal Processing (Special Issue on Wireless Location Technologies and Applications)

Gezici,S., Sahinoglu, Z., Molisch, A., Kobayashi, h. \& Poor, H. (2008). Two-step time of arrival estimation for pulse based ultra-wideband systems, In: EURASIP Journal on Advances in Signal Processing, vol. 2008, Article ID 529134, 11 pages

Gezici, S., Tian, Z, Giannakis, G., Kobayashi, H., Molisch, A.F., Poor, H. \& Sahinoglu, Z. (2005). localization via ultra-wideband radios: a look at positioning aspects for future sensor networks, In: IEEE signal processing Magazine, pp. (22:70-84)

Guvenc, I. \& Sahinoglu, Z. (2005). TOA estimation with different IR-UWB transceiver types, In: Proc. IEEE Int. Conf. UWB, pp. (426-431), Zurich, Switzerland

Guvenc,I.; Sahinoglu, Z.; Molisch,A. \& Orlik, P. (2005). Non-coherent TOA estimation in IR-UWB systems with different signal waveforms, In: Proc. IEEE Int. Workshop on Ultrawideband Networks (UWBNETS), pp. (245-251)

Guvenc, I. \& Sahingolu, Z. (2005). Threshold-based TOA estimation for impulse radio UWB systems, In: Proc. IEEE Int. Conf. UWB, pp. (420-425), Zurich, Switzerland

Guvenc, I. \& Sahinoglu, Z. (2005). Threshold selection for UWB ToA estimation based on kurtosis analysis, In: IEEE Commun. Lett., pp. (1025-1027), Vol. 9, No. 12

Guvenc, I., Shahinoglu, Z. \& Orlik, P. (2006). TOA Estimation for IR-UWB Systems with Difference transceiver Types, In: IEEE Trans. on Microwave Theory and Techniques, Vol. 54 , No. 4

Guvenc, I., Gezici, S. \& Sahinoglu, Z. (2008). Ultra-wideband range estimation: Theoretical limits and practical algorithms, In: Proc. IEEE International Conference on Ultra-Wideband (ICUWB 2008), pp. (93-96), Hannover, Germany

Haneda, H., Takizawa, K., Takada, J., Dashti, M. \& Vainikainen, P. (2009). Performance Evaluation of Threshold-Based UWB Ranging Methods-Leading Edge vs. Search Back-, In: 3rd European Conference on Antennas and Propagation, pp. (3673-3677)

Haneda, K., Takada, J., Takizawa K. (2007). Ultra Wideband Path Loss Modelling in a Line-of-Sight Office Environment. 2nd European Conference on Antennas and Propagation (EuCAP 2007), Nov. 2007 (Edinburgh, UK).

IEEE Std. (2007). Wireless Medium Access Control (MAC) and Physical Layer (PHY) Specifications for Low-Rate Wireless Personal Area Networks(WPANs), In: IEEE Std 802.15.4a-2007, pp. (81-83)

Jourdan, D. (2006). Wireless Sensor Network Planning with Application to UWB Localization in GPS-Denied Environments, In: Doctoral Thesis at Massachusetts Institute of Technology

Lee, J. \& Scholtz, R. (2002). Ranging in a dense multipath environment using an UWB radio link, In: IEEE Journal on selected Areas in Communications, pp. (20(9):1677-1683) 
Low, Z., Cheong, J., Law, C., Ng, W., \& Lee, Y. (2005). Pulse detection algorithm for Line-of-Sight (LoS) UWB ranging application, In: IEEE Antennas and Wireless Propagation Letters, pp. (4:63-67)

Molisch, A.F., Balakrishnan, v, Cassioli, D., Chong, C.C., Emami, S., Fort, A., Karedal, J., Kunisch, J., Schantz, H., Schuster, U. \& Siwiak, K. (2004). IEEE 802.15.4a channel model - final report, IEEE 802.15 WPAN Low Rate Alternative PHY Task Group 4a(TG4a), In: Tech. Rep.

Sahinoglu, Z., Gezici, S. \& Guvenc, I. (2003). Ultra-wideband Positioning Systems: Theoretical Limits, Ranging Algorithms, and Protocols, In: Cambridge university press

Scholtz , R. \& Lee, J. (2002). Problems in modeling UWB channels, In: Proc. IEEE Asilomar Conf. Signals, Syst. Computers, pp. (706-711), , vol. 1, Pacific Grove, CA

Win, M. \& Scholtz, R. (1998). On the robustness of ultra-wide bandwidth signals in dense multipath environments, In: IEEE Commun. Lett., pp. (2(2):51-53)

Win, M. \& Scholtz, R. (2002). Characterization of ultra-wide bandwidth wireless indoor communications channel: A communication theoretic view, In: IEEE J. Select. Areas Commun., pp. (20(9):1613-1627)

$\mathrm{Xu}, \mathrm{C}$. \& Law, C. (2008). Delay-dependent threshold selection for UWB ToA estimation, In: IEEE Communication letters, pp. (380-382), Vol. 12, No.5

Yang, L. \& Giannakis, G. (2004). Ultra-wideband communications: An idea whose time has come, In: IEEE Sig. Processing Mag., pp. (26-54), vol. 21, no. 6

Yang, L. \& Giannakis, G. (2005). Timing ultra-wideband signals with dirty templates, In: IEEE Trans. Commun., pp. (1952-1963), vol. 53, no. 11 


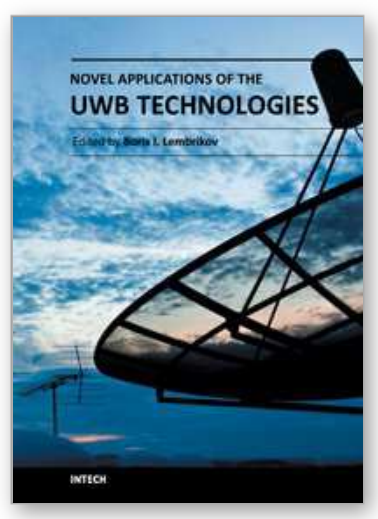

\author{
Novel Applications of the UWB Technologies \\ Edited by Dr. Boris Lembrikov
}

ISBN 978-953-307-324-8

Hard cover, 440 pages

Publisher InTech

Published online 01, August, 2011

Published in print edition August, 2011

Ultra wideband (UWB) communication systems are characterized by high data rates, low cost, multipath immunity, and low power transmission. In 2002, the Federal Communication Commission (FCC) legalized low power UWB emission between $3.1 \mathrm{GHz}$ and $10.6 \mathrm{GHz}$ for indoor communication devices stimulating rapid development of UWB technologies and applications. The proposed book Novel Applications of the UWB Technologies consists of 5 parts and 20 chapters concerning the general problems of UWB communication systems, and novel UWB applications in personal area networks (PANs), medicine, radars and localization systems. The book will be interesting for engineers and researchers occupied in the field of UWB technology.

\title{
How to reference
}

In order to correctly reference this scholarly work, feel free to copy and paste the following:

Marzieh Dashti, Mir Ghoraishi, Katsuyuki Haneda and Jun-ichi Takada (2011). High-Precision Time-of-Arrival Estimation for UWB Localizers in Indoor Multipath Channels, Novel Applications of the UWB Technologies, Dr. Boris Lembrikov (Ed.), ISBN: 978-953-307-324-8, InTech, Available from:

http://www.intechopen.com/books/novel-applications-of-the-uwb-technologies/high-precision-time-of-arrivalestimation-for-uwb-localizers-in-indoor-multipath-channels

\section{INTECH}

open science | open minds

\section{InTech Europe}

University Campus STeP Ri

Slavka Krautzeka 83/A

51000 Rijeka, Croatia

Phone: +385 (51) 770447

Fax: +385 (51) 686166

www.intechopen.com

\section{InTech China}

Unit 405, Office Block, Hotel Equatorial Shanghai

No.65, Yan An Road (West), Shanghai, 200040, China

中国上海市延安西路65号上海国际贵都大饭店办公楼 405 单元

Phone: +86-21-62489820

Fax: +86-21-62489821 
(C) 2011 The Author(s). Licensee IntechOpen. This chapter is distributed under the terms of the Creative Commons Attribution-NonCommercialShareAlike-3.0 License, which permits use, distribution and reproduction for non-commercial purposes, provided the original is properly cited and derivative works building on this content are distributed under the same license. 